\title{
Corela
}

Cognition, représentation, langage

2-1 | 2004

Vol. $2, n^{\circ} 1$

\section{Quelques problèmes métalinguistiques en phonologie de l'anglais}

\author{
Nicolas Ballier
}

\section{(2) OpenEdition}

Journals

Édition électronique

URL : http://journals.openedition.org/corela/594

DOI : $10.4000 /$ corela.594

ISSN : 1638-573X

Éditeur

Cercle linguistique du Centre et de l'Ouest - CerLICO

Référence électronique

Nicolas Ballier, «Quelques problèmes métalinguistiques en phonologie de l'anglais », Corela [En ligne], 2-1 | 2004, mis en ligne le 15 juin 2004, consulté le 01 mai 2019. URL : http://journals.openedition.org/ corela/594; DOI : 10.4000/corela.594

Ce document a été généré automatiquement le 1 mai 2019.

\section{(c) (i) (2)(2)}

Corela - cognition, représentation, langage est mis à disposition selon les termes de la licence Creative Commons Attribution - Pas d'Utilisation Commerciale - Partage dans les Mêmes Conditions 4.0 International. 


\title{
Quelques problèmes métalinguistiques en phonologie de l'anglais
}

\author{
Nicolas Ballier
}

\section{Introduction}

1 Cette contribution a pour corpus privilégié la métalangue des enseignants et des manuels français de phonologie anglaise ${ }^{1}$, et, ponctuellement, des étudiants. A partir de quelques problèmes concrets, nous envisagerons les questions théoriques propres au rapport entre l'objet langue envisagé sous sa matérialité sonore et les discours construits pour en rendre compte. Certaines des questions ici soulevées seront plus spécifiques aux anglicistes, en particulier le statut de la variété décrite et surtout la place prépondérante que l'accent lexical ou les relations grapho-phonématiques (entre écriture et prononciation) occupent dans la description de cette langue en France ${ }^{2}$. Pour autant, quelques-unes de ces questions ne se limiteront pas au seul enseignement de l'anglais aux francophones.

2 Ces questions de représentations métalinguistiques liées à la phonologie de l'anglais seront étudiées ici exclusivement au plan segmental ${ }^{3}$. Certaines des problématiques seraient transversales et récurrentes pour le suprasegmental (accentuation et intonation), en particulier, la traduction, pour ne pas dire l'incarnation, des choix théoriques dans les conventions notationnelles et la question des formes citationnelles des paradigmes. Au niveau donc du segmental (de la représentation ${ }^{4}$ des phonèmes), on montrera que, pour les "apprenants», l'activité de représentation passe par une déconstruction des représentations existantes, y compris les plus fondatrices (représentation de ce qu'est une consonne, une voyelle ou une syllabe). Elle est de plus déplacée (plus que résolue) en phonologie par la transcription phonétique, dont on montrera quelques effets, en particulier s'agissant des symboles phonétiques comme système sémiotique dont la logique s'écarte des fonctionnements habituels des systèmes 
d'écriture. Au-delà, nous voudrions questionner la possibilité offerte en phonologie de reproduire le signal, le fait de langue dans sa matérialité phonétique, envisageant ainsi quelques conséquences théoriques de la reproductibilité technique de ce que le phonologue cherche à décrire. En deçà, nous nous devons d'évoquer les incidences métalinguistiques de la variété décrite.

\section{L'objet décrit et sa variation}

3 La question de la variabilité de l'objet décrit est sans doute déterminante pour la langue anglaise. Dans un monde où les locuteurs de l'anglais langue seconde sont numériquement majoritaires, le système décrit est par nature un "construit" et susceptible d'évoluer. L'aspect métalinguistique de cette question tient aussi selon nous en ce qu'un métadiscours peut ne pas manifester les propriétés qu'il est censé décrire. Dans l'absolu, c'est aussi le statut d'un métadiscours explicatif en français sur la grammaire anglaise, où par exemple nul auxiliaire modal ne viendra illustrer un cours sur les modaux. Au plan phonologique, l'impression résultante est plus complexe, puisque deux occurrences " contradictoires ", cohérentes dans leur système (d'un côté l'idiolecte de l'enseignant, par exemple un lecteur anglophone et de l'autre les propriétés du système décrit), ne sont pas nécessairement superposables. On ne préjugera pas ici de la norme qu'il convient d'enseigner, c'est-à-dire du système phonologique que l'on souhaite transmettre. Nous indiquons sommairement la conséquence pratique et théorique de ces possibilités de décalages et le rôle dévolu à la variété de référence.

4 La question de la «norme » décrite ne sera donc pas résolue ici, mais elle ne sera pas oubliée pour autant, car il arrive que les cours de phonétique soient faits par les lecteurs anglophones dont l'inventaire phonologique s'écarte notablement des documents qu'ils diffusent en cours. D'expérience, celle-ci transfère, du moins au début, l'insécurité linguistique des enseignés aux enseignants et matérialise sous forme de contradiction ( «je ne prononce pas ce que j'enseigne ») certaines des difficultés notionnelles liées au statut théorique du phonème, à la transcription large et aux inventaires vocaliques. Nous y reviendrons, mais les conditions pratiques d'enseignement de la phonétique illustrent, de fait, le décalage entre le type et l'occurrence. Ce que, littéralement, on donne à entendre n'est pas rigoureusement superposable à ce que l'on cherche à donner à comprendre. Pour prendre un exemple caricatural, certains lecteurs anglophones doivent expliquer que le « $\mathrm{r}$ » dans les variétés non-rhotiques ne se prononce pas en position postvocalique quand eux-mêmes le prononcent spontanément. Plus généralement, leur système vocalique n'est pas rigoureusement celui de la variété de référence. Nous parlerons là d'une opacification du type sous l'occurrence dans le moment de la confrontation au "réel linguistique ", c'est-à-dire à la multiplicité des réalisations phonétiques.

5 Nous ferons ici l'hypothèse raisonnable que ce système phonologique enseigné, la «norme » (Standard British English en général, quelle que soit sa dénomination) sert de cadre de référence. L'inventaire vocalique (c'est le plus problématique) de la norme fonctionne comme schème de variation. Il s'agit, là encore, de «construire du déformable » (Culioli), cette fois-ci en permettant l'intelligibilité mutuelle d'un inventaire phonémique anglophone à un autre. A des degrés divers de technicité, on doit pouvoir rendre compte, par exemple, de réalisations homophoniques pour un locuteur d'une variété donnée alors que la graphie et la bonne compréhension des énoncés attesteraient 
d'une paire minimale dans la variété de référence. On pourrait dire que l'objectif final est la mise en place d'un système de conversion d'un système phonémique à un autre ( mapping), ce qui induit des choix quant à la variété de référence, véritable passerelle entre les variétés d'anglais possibles. D'une certaine manière, la variété de référence vaut moins pour elle-même que pour les opérations de conversions qu'elle autorise dans l'intelligibilité mutuelle entre locuteurs de variétés différentes. En résumé, la question de la variété décrite (et par ailleurs de la variété parlée par l'enseignant) manifeste l'écart possible entre réalisations et transcriptions et pose de manière cruciale pour la linguistique anglaise le rapport entre le type et l'occurrence. La variété de référence n'est pas qu'une question sociolinguistique; c'est un filtre, au moins perceptuel, à l'appréhension du segmental.

6 C'est dans l'analyse du segmental, a fortiori dans la tradition française de la graphophonématique où le segment phonétique s'appréhende aussi à partir de correspondances graphiques et de symboles phonétiques, que se nouent selon nous bien des problèmes de représentations ainsi que des enchevêtrements de niveaux d'analyse, de systèmes sémiotiques, voire de procédures cognitives. Chemin faisant, nous illustrerons deux thèses solidaires. Premièrement les métadiscours sont susceptibles de produire des artefacts de présentation, voire des équivoques représentationnelles. Deuxièmement, nous faisons, après d'autres, l'hypothèse que ces représentations erronées sont aussi des symptômes potentiels des difficultés posées par la théorie, s'ajoutant en quelque sorte à "l'insu" que génère chaque théorie linguistique ${ }^{5}$. En clair, les équivoques représentationnelles sont vues comme un moyen d'accès à une partie du dispositif métalinguistique, un peu à la manière de la grammaire des fautes pour la grammaire. On cherchera davantage à fonder en raison certaines des équivoques représentationnelles qu'à en théoriser la succession au cours des apprentissages. Nous essayons ici d'en sérier certains aspects, en partant du choix même de commencer par le segmental.

\section{Par où commencer?}

7 L'appréhension générale de la phonologie comme champ d'étude, la compréhension de ce qu'est le système phonologique et les procédés d'analyse en vigueur sont conditionnées par la démarche adoptée. Statistiquement ${ }^{6}$, la démarche la plus rationnelle dans l'ordre de préséance des phénomènes phonologiques, du placement du noyau intonatif à la réalisation vocalique, (top down) est minoritaire dans les manuels, qui privilégient un ordre plus pédagogique, des unités minimales aux macro-unités (bottom-up). Cette organisation de l'exposé des connaissances met au jour dès le départ l'extrême solidarité des concepts phonologiques, au risque de la circularité dans les explications (entre la paire minimale, le phonème, la distinction pertinente). L'autre effet pervers de cette approche est sans doute de mobiliser les énergies sur des problèmes de réalisation segmentale, sans toujours faire nettement percevoir que ceux-ci sont conditionnés par des placements accentuels qui sont de toute façon bien plus déterminants pour l'intelligibilité d'un mot pour une oreille anglophone.

8 En d'autres termes, nous tenons que l'accès à un domaine de connaissance est tributaire de son mode d'exposition. Cette problématique (des effets d'exposition, des choix théoriques de présentations) nous semble indissociable des questions métalinguistiques. Plus précisément, elle relève pour nous d'un substrat théorique du métadiscursif qui se résume ainsi : «par où commencer? » Nous étaierons notre thèse "constructiviste » à 
partir des effets de la paire minimale. Par «effets », nous entendons le décalage entre deux représentations, que nous tenons pour un artefact des métadiscours comme corps de discours produits sur ce que l'on souhaite décrire.

Toute question de cours ou de réflexion qui dévie sur une redéfinition par l'étudiant de la paire minimale conduit à des exemples tous construits pour le même modèle. Au point que l'un des exemples donnés en cours finit par faire oublier tous les autres et surtout autorise potentiellement toutes les extrapolations à partir des propriétés vérifiées dans l'exemple, lesquelles deviennent alors des conditions nécessaires et suffisantes. Entre autres, la répétition d'exemples à consonne initiale identique occulte les distinctions entre le statut de phonème et les contraintes distributionnelles (que la nasale vélaire / g / ne soit jamais en position initiale ne l'empêche pas d'être un phonème de l'anglais moderne). Pour l'anglais, l'exemple typique des paires minimales est informé par les structures phonologiques. Pour les voyelles brèves, c'est un mot en CVC (type <sit> ou <sat>) qui oppose les consonnes initiales ou la voyelle médiane, nécessairement brève. Il illustre également un gabarit, une structure de syllabe, autant que la seule distinctivité de la paire minimale. A titre de viatique, à défaut de panacée, nous formulons deux suggestions. Premièrement, décliner les différents gabarits possibles, notamment les dissyllabiques. Deuxièmement, la paire minimale n'est pas nécessairement obscène, mais elle frappe davantage les esprits lorsqu'elle l'est ${ }^{7}$. Lorsque tel est le cas, l'étudiant est obligé de la reformuler en raison du tabou qui pèse sur ce que l'on peut écrire dans une copie d'examen. Le moindre des malentendus n'est pas que ce qui est une procédure d'investigation d'un système phonologique est perçu comme un exemple d'opposition binaire entre deux «mots». L'occurrence vaut pour le type, l'exemple pour la méthode d'analyse et le lexical pour le phonologique. Dans ce cas précis des paires minimales, la représentation du concept linguistique est obérée par le mode d'exposition. Il y a bien des artefacts de présentation, en un sens des effets de paradigmes.

\section{Le primat graphocentriste}

10 Si ce réseau conceptuel fondateur de la phonologie peut être tributaire de son mode d'exposition, d'autres notions de base de la phonologie doivent s'affronter au graphocentrisme. Plusieurs faits peuvent illustrer les difficultés représentationnelles liées à l'importance de l'écrit dans le système scolaire français. Nous en donnons quelques exemples, s'agissant des métatermes phonologiques, avant de suggérer qu'elles s'incarnent également dans une confusion récurrente dans les transcriptions phonétiques.

$11 \mathrm{Au}$ début était l'écrit. Les notions mêmes de «voyelles » et de « consonnes » renvoient à de l'écrit ${ }^{8}$. Ces notions centrales de la phonologie ont pour nom des termes associés depuis longtemps à tout autre chose dans l'esprit des étudiants, ce qui suppose une déconstruction voire un désapprentissage complet de leurs représentations scolaires. L'inventaire des voyelles en phonologie n'est pas $\langle a\rangle,\langle e\rangle,\langle i\rangle,\langle 0\rangle,\langle\mathrm{u}\rangle$ et $\langle y\rangle$. Les soucis du phonologue ne s'arrêtent pas là. Une fois que l'on a procédé à cette mise au point, reste à s'assurer de l'autonomie des représentations des voyelles et des consonnes. Pour ces dernières, le phonème tend, plus ou moins consciemment, à être posé dans l'esprit des étudiants comme étant ce que la lettre note (sans compter que bien des symboles phonétiques correspondants sont les lettres de l'alphabet romain). Ce donné se complique d'autant que les étudiants francophones reçoivent souvent un enseignement inspiré par 
l'école de Guierre, qui précisément se fonde sur la graphie pour prédire des placements accentuels et des réalisations phonématiques. L'ambiguité dans la métalangue de «voyelle» ou «consonne » met au jour ces problèmes de représentation conflictuelle entre héritage scolaire graphique et conceptualisation phonologique. Malheureusement, la convention notationnelle traditionnelle perpétue cette ambiguïté en choisissant de représenter la consonne par $\mathrm{C}$ et la voyelle par $\mathrm{V}$, sans toujours distinguer entre $<\mathrm{C}>$ et $\mathrm{C}^{9}$, c'est-à-dire entre la consonne graphique et la consonne phonologique. Cette ambiguïté est parfois à l'oeuvre dans la formulation des règles grapho-phonématiques dans les manuels.

12 La démonstration peut valoir aussi pour la syllabe. Sa représentation en phonologie est moins consensuelle, mais le décalage demeure avec la représentation héritée de l'école. En caricaturant un peu, la syllabation y est réduite à l'activité qui consiste à placer le tiret entre les bonnes lettres pour aller à la ligne. L'image est plus complexe en phonologie. Non seulement les découpages des mots en syllabes des mêmes mots est différent en français et en anglais (trois syllabes contre quatre pour millionaire, à titre d'exemple), mais ce que l'on peut accepter comme syllabe change radicalement. Ainsi, pour des francophones, il ne faut pas négliger l'effort cognitif que constitue l'existence en anglais de syllabes sans voyelles, comme dans le cas des consonnes syllabiques (ainsi dans la syllabe finale de simple $e^{10}$ ).

13 L'in-scription dans l'écrit des représentations langagières fonctionne comme autant de résistances à l'appréhension des concepts phonologiques. La question est complexe, puisque si la graphie (la représentation orthographique du mot) n'est pas indispensable à la conceptualisation phonologique, il faut bien passer par l'écrit pour codifier une représentation (qu'il s'agisse d'une arborescence de traits, d'une structure syllabique ou d'une réalisation phonologique). Néanmoins, il nous semble que le rapport à l'écrit fonctionne trop souvent comme une «béquille » de la représentation, à situer certes dans un système scolaire et universitaire donné, mais dont les effets ne sont pas toujours suffisamment mesurés. Même lorsqu'il s'agit d'un recours à l'écrit "légitime ", dans le cadre d'une transcription phonologique, le primat graphocentriste se fait sentir. Nous voulons le montrer à partir de l'exemple des fricatives dentales, prises comme équivoque représentationnelle révélatrice d'un rapport au symbole phonétique qui ne s'affranchit pas d'un rapport à la lettre.

Il s'agit de rendre compte de la confusion fréquente, y compris chez les agrégatifs, entre les symboles phonétiques notant la sourde et la sonore de la fricative interdentale ${ }^{11}$. Certes la cause est multifactorielle. Les phonèmes n'existent pas en français et les symboles phonétiques ne sont pas inspirés par des caractères de l'alphabet romain. Entre autres, le symbole est muet et rien ne permet de mémoriser a priori le caractère sourd ou sonore de ce qu'il représente. Pire, s'illustre ici un phénomène bien connu où un distinguo est mémorisé en intension mais pas en extension. L'étudiant sait qu'il y a une opposition structurale, il y a une sourde et une sonore, mais ne sait pas les identifier. La distinctivité trouve sans doute un de ses effets pervers dans cette confusion entre sonore et sourde. Les transcriptions erronées révèlent que l'identification avec la fricative dentale est correcte, mais que le caractère sourd et sonore ne l'est pas ; ce qui est aussi à rapprocher de la réticence des étudiants à constater la vibration des cordes vocales d'un simple geste en plaçant la main sur la pomme d'Adam. Alors que la phonétique est aussi affaire de tuyauterie, la réticence du rapport au corps est notoire, mais elle prive l'esprit de l'interface phonétique / phonologie en son versant articulatoire dans ce qui n'est autre 
qu'une «technique du corps » que n'aurait pas désavouée Marcel Mauss. Ceci relève au fond d'une pratique « silencieuse » de la lecture des transcriptions phonétiques, qui est de fait un obstacle à la prise de conscience de ces phénomènes de voisement. Les équivoques représentationnelles (un symbole pour un autre, mais du même coup une interchangeabilité du trait [+/- voisé]) manifestent bien des interférences de type "graphique " au sens où un symbole phonétique est "silencieusement" associé à un digraphe indépendamment de toute réalisation phonétique. Il nous semble que c'est l'unité graphique $d u<t h>$, toujours en arrière plan, qui autorise ces flottements, voire cette interchangeabilité entre les symboles phonétiques. Pour couronner le tout, le TH- a maintenant un statut sémantique (voire morphologique) ${ }^{12}$ dans la vulgate grammaticale de l'étudiant de Licence. Derrière les erreurs d'attribution de symbole peut se lire un empilement de représentations (graphiques, étymologiques voire morphologique) et de désignations (prononcer « thé, hache ») dont l'incarnation est graphémique $<$ th $>^{13}$.

La métalangue des apprenants trahit également ce primat graphique dans la représentation phonologique. Dans les commentaires d'étudiants, la persistance d'expressions telle que « raccourcir la voyelle » (plutôt que «abréger ») ne participe pas seulement d'une simplification de la métalangue ; c'est sans doute aussi un reflet de la relation iconique au diacritique (le signe de la longueur est un attribut que l'on peut perdre) et donc d'une représentation "graphique» du phonologique. Or, tant qu'à construire une représentation iconique de la langue, autant mettre en rapport la longueur de la voyelle avec des spectrogrammes de longueur variable. Consciemment ou non, le segmental est associé à de l'écrit, qu'il s'agisse de graphie ou de transcription phonétique. Se dessine ici un graphocentrisme paradoxal où la transcription phonologique est au centre, soit un écrit qui rend compte de l'oral mais qui ne s'y réduit pas (sans compter que bien des représentations phonologiques sont abstraites et ne sont pas représentées dans les transcriptions). Tout ceci revient à rappeler, truisme lourd d'implications, que l'activité de transcription fait passer les questions phonologiques par le filtre de l'écrit ${ }^{14}$. C'est à un examen de quelques-unes de ces implications théoriques que nous nous attelons maintenant, en particulier quant au statut métalinguistique à accorder aux transcriptions phonétiques et aux conventions notationnelles.

En toute logique, il convient de se demander dans quelle mesure les représentations phonologiques peuvent s'émanciper de l'écrit, ce qui impose de questionner les types de métadiscours construits pour rendre compte des phonèmes. Nous abordons ce vaste problème sous trois aspects. Primo, comment prendre en compte l'influence de l'écrit, dont la représentation associée au phonème n'est sans doute pas uniforme dans le temps? Secundo, dans quelle mesure l'abstraction du phonème se laisse-t-elle prendre dans le symbole qui le représente? Tertio, quels sont les moyens d'accès métadiscursifs aux phonèmes de l'anglais?

\section{Le phonème ou la sédimentation des représentations}

17 A grands traits, l'hypothèse nous paraît être la suivante. S'il n'est pas nécessaire de savoir écrire pour parler, l'écrit n'est pas une condition nécessaire à la représentation des phonèmes. En revanche, la connaissance de l'écrit joue un rôle dans la représentation des phonèmes. Ceci revient potentiellement à admettre que la représentation des phonèmes 
n'est pas uniforme ni pour les individus, ni dans le temps. Une périodisation fictionnelle, où l'ontogenèse récapitule la phylogenèse, pourrait être celle-ci :

- représentation phonologique : l'enfant sait parler, acquérir un système phonologique sans nécessairement savoir le décrire. Les phonèmes, toutes les langues sans écriture le savent, n'ont pas besoin d'écrit pour exister. L'apprenti locuteur a pleinement conscience de ce qu'il peut mal prononcer certains « sons » et sait quand on se moque de lui.

- représentation graphique : l'élève sait qu'il y a des sons et des lettres pour les écrire. Les listes d'homophones et les exceptions orthographiques construisent comme des protoreprésentations du phonème comme ce qui sert à subsumer cette variation graphémique.

- représentation grapho-phonologique: l'étudiant assiste au cours de phonétique. Le phonème est ce que la lettre note. D'ailleurs, c'est souvent une lettre qui sert de symbole API.

- représentation (méta-)phonologique: le linguiste sait que la correspondance graphie/ phonie n'est pas biunivoque et qu'il existe une spécificité des séquences phonématiques indépendamment de la graphie.

18 Le problème de ces représentations divergentes est bien d'ordre métalinguistique en ce que, d'une part, des termes identiques renvoient à des représentations différentes et, d'autre part, des termes différents renvoient manifestement à des complexités variables de représentation (lettres vs graphèmes / lettres vs trigraphes ou digraphes). Notre fiction est commode puisqu'elle installe le phonologue dans le fauteuil de l'histoire, où son point de vue est le point d'arrivée de cette généalogie du concept. De ces conceptualisations successives des métatermes, un peu sur le mode des strates d'interlangue, on peut être tenté de considérer que seule la représentation du phonologue est légitime et complète ; le reste n'étant que des proto-représentations, des représentations «épilinguistiques» (Culioli). Une autre hypothèse est néanmoins possible: dans l'activité cognitive des sujets, la représentation du concept (ici le phonème) ne serait pas uniforme. Non seulement le concept linguistique est labile dans le travail du concept au fil de l'analyse, mais il ne serait pas stable dans les représentations que l'on peut en formuler et notamment selon les locuteurs. Notre fiction étaye cette hypothèse dans la diachronie des sujets, mais elle doit avoir aussi sa traduction au plan synchronique dans la comparaison selon les langues du rôle joué par la conscience de l'orthographe et donc de la graphie. Il ne s'agit pas de dire que les langues sans écriture n'ont pas de phonèmes. Il y a à penser l'influence de l'orthographe dans les langues qui en ont la tradition, ce qui conduit, entre autres, à s'interroger sur les représentations associées chez les locuteurs aux séquences graphiques et contribuera à fonder en raison le primat de l'écrit dans les analyses grapho-phonématiques de l'école de Guierre ${ }^{15}$.

19 L'analyse phonologique n'est pas antinomique de la graphie, mais conduit à interroger certaines pratiques, conscientes ou non. Cela passe par la prise en compte des sédimentations de représentations, décrites par Anne Trévise pour la grammaire anglaise, où la connaissance universitaire de type métalinguistique se heurte aux savoirs scolaires hérités. Au plan phonologique, il nous semble que la dimension scripturale intervient, pour la transcription et les symboles phonétiques mais également dans la manière dont les phonèmes sont présentés. 


\section{L'abstraction du phonème}

20 brute. Ce que l'on enseigne en cours de phonétique / phonologie est également le résultat d'une abstraction d'un donné plus ou moins brut. Cela vaut selon nous pour le concept de phonème $\mathrm{e}^{16}$, ce que met en évidence un questionnement du symbole phonétique qu'on lui associe ou de sa forme citationnelle.

de cette entité théorique, le phonème en tant que tel ne se prononce pas. Abstraction (classe de tous les allophones qu'il subsume pour certains), le phonème est tout au plus désigné par ce qui lui tient lieu de forme désignationnelle. Au mieux peut-on y référer, notamment par le symbole qui le note. Ce paradoxe de l'impossibilité de la prononciation du symbole est au cœur des avertissements de l'enregistrement par John Wells et Jill House pour The Sounds of the IPA, enregistrement d'une lecture, pour ne pas dire d'une interprétation, des symboles de l'API. Les consonnes sont prononcées en position isolée ou intervocalique (couvrant ainsi le spectre des allophones types) mais l'on peut très bien ne pas reconnaitre ce qui est censé être un exemple possible d'un phonème de sa langue. Tout symbole phonétique est en quelque sorte muet et n'a pas de représentation associée stable (mais un faisceau de réalisations ou phones). pris par convention comme l'étendard de sa classe. Or ce choix du paradigme désignationnel ou de sa forme citationnelle ne va pas de soi. Pour prendre un exemple extrême, quel sens donner à une étiquette comme /t/ quand un locuteur réalise systématiquement un coup de glotte ? La désignation même du phonème par un symbole phonétique ne va pas de soi. Pourquoi ne pas utiliser [ $\mathrm{t}]$, après tout plus fréquent pour l'anglais américain, pour noter la latérale alvéolaire /1/ ? Il est un écart entre forme désignationnelle et ensemble des réalisations concrètes qui affecte bien des unités minimales. La seule différence, mais elle est de taille, est qu'au plan phonologique, cette différence est matérialisée dans le commentaire métalinguistique. Dans le moment de l'articulation d'une prononciation effective, c'est un phone que l'on donne à entendre en guise de phonème. Nous retrouverons ce paradoxe performatif à propos des formes faibles. Une comparaison rapide du phonème avec ses allophones et du morphème avec ses allomorphes peut contribuer à affiner cette spécificité du phonologique qui travaille sur de l'allophonie. $\{-S\}$ ne se donne pas pour clé de tous les allomorphes du pluriel possibles, mais bien comme un «symbole» du pluriel. L'écrit fait qu'il est d'ailleurs isolable en corpus, attestable dans les néologismes, voire qu'il peut apparaitre comme non-marqué (par rapport à children ou à feet), ce que l'on demande souvent aux formes citationnelles, et il est statistiquement motivé. Rien de tel en phonologie où le symbole ne note pas nécessairement la réalisation la plus fréquente (loin s'en faut si l'on pense à $/ \mathrm{r} /$ ). Le statut d'observable du phonème est plus complexe, comme le révèlent également les problèmes posés par l'exposé des modes d'accès aux phonèmes de l'anglais.

\section{Vicissitudes métadiscursives}

Explorons quelques difficultés liées à ces réponses qu'on voudrait d'une simplicité biblique. Comment donne-t-on accès aux phénomènes langagiers dont on cherche à rendre compte? Nous voudrions soulever quelques-unes des difficultés de ce que nous 
tenons comme des modes d'accès métadiscursifs aux phonèmes. Ce sont les voyelles, et en particulier les voyelles centrales, que nous prendrons comme exemple ${ }^{17}$.

\section{Perception auditive}

Le crible phonologique fait que les locuteurs sont "sourds " aux phonèmes qui ne sont pas ceux de leur langue maternelle. On est de plus tributaire de la variété d'anglais du locuteur et, de toute façon, on ne donnera à entendre que des phones. Le problème théorique reste entier. (Le phonème ne se prononce pas, le symbole est muet).

\section{Représentations visuelles sur le trapèze de Jones}

Rappelons que «le champ de dispersion du phonème" (Martinet) interdit une représentation stricte sur le trapèze de Jones en un point unique. C'est une zone qui matérialise les occurrences possibles des phonèmes, de sorte que les zones peuvent se recouvrir, notamment pour les voyelles centrales ${ }^{18}$.

\section{Représentation des dictionnaires (la médiation par l'orthographe)}

Une compilation des principaux exemples donnés dans les dictionnaires unilingues pour anglophones afin d'illustrer les phonèmes réserve de nombreuses surprises aux étudiants de DEUG (cf liste en annexe). Ainsi pour / $\mathrm{a} /$, c'est $\mathrm{SON}^{19}$ qui tient lieu d'illustration. L'exemple est une exception historique aux correspondances grapho-phonématiques enseignées par ailleurs. Au voisinage des lettres à jambages, mother, another ne sont pas "spontanément » associés à la voyelle de BUS pour un francophone et cela perturbe la perception des <0> interconsonantiques. Ceci revient à admettre que les représentations prototypiques des phonèmes, à supposer qu'elles soient possibles, associées aux mots ne sont pas nécessairement les mêmes pour les francophones et pour les anglophones.

Plus qu'ailleurs (dans la description de la langue), le choix de l'exemple informe la représentation du paradigme qu'il explicite, voire, dans certains cas, qu'il désigne (lorsque l'exemple sert à désigner la règle ou la classe de mots). La charge symbolique d'un mot et sa place dans l'apprentissage ne coïncident pas nécessairement. Pas davantage que sa fréquence d'emploi ne permet de préjuger du caractère prototypique des relations graphie-phonie qu'il manifeste. Ainsi BLOOD, donné comme exemple de / $\mathrm{A}$ /, est une réalisation exceptionnelle du digraphe <0o>. Il peut y avoir un décalage entre la forme la plus fréquente et celle donnée en exemple. Or, le statut sémantique d'un terme comme <blood> n'est pas comparable à la pertinence dans la fréquence de la réalisation de son digraphe vocalique. On voit les risques de disproportion entre le poids sémantique du lexème et le rôle d'exemple qui lui est dévolu en phonologie. L'exemple a non seulement un statut mais une valeur mnésique, une charge symbolique, il entre dans un réseau de connotations (telles SUN et SON comme représentants de / $\mathrm{A} /$ ). On ne saurait tenir jusqu'au bout que toutes les équivoques représentationnelles sont un artefact de la métalangue. Néanmoins, se repose ici la question du statut de l'exemplum (cf. Milner 1989) et des effets d'optique qu'il induit. Ce n'est pas un métaterme strict, mais une composante essentielle du métadiscours. Il relève pour nous de la métalangue au sens large et a un statut important comme schème d'accès de l'occurrence au type. 


\section{Fossilisation des paradigmes}

Il y a un risque inhérent à se servir de mots peut-être déjà mal acquis, ou de formes fossilisées mais associées à des représentations erronées (telle une réalisation de her qui rimerait avec peur).

\section{Symbolisation}

La transcription phonétique (transcription comme activité) met en jeu ce qui nous apparait être son corollaire, la captation de la représentation du phonème à partir de la symbolisation phonétique. La médiation par la simple reproduction du signal ne constitue pas une représentation quand elle n'est pas associée à une représentation graphique, instrument d'une réelle conceptualisation. Ceci n'offre aucune garantie quant à la question de savoir ce à quoi le symbole est associé, mais cela impose un détour par le statut métalinguistique de la transcription.

\section{Du statut métadiscurif de la transcription}

Le métaterme est lui-même ambigu. C'est un terme processif ${ }^{20}$ qui renvoie potentiellement à un processus (la pratique de la transcription) ou à son résultat (la transcription comme tracé graphique). Dans l'ensemble, la transcription comme résultat ne règle rien de manière définitive et n'épuise pas le "réel» phonétique dans sa description. Elle se contente de révéler l'existence de la transcription comme processus, comme activité, qui est un autre nom de la possibilité de construire des métadiscours. De ce point de vue, la transcription est une propriété, le fait de pouvoir être transcrit, le «transcriptible» en quelque sorte. L'essentiel nous paraît résider dans cette transposabilité d'un système de conventions et de valeurs à un autre système de conventions et de valeurs. La transcription comme activité est une application (au sens mathématique de la relation d'un ensemble à un autre) entre, potentiellement :

- le système graphique et l'alphabet phonétique international tel qu'il est utilisé pour la description de l'anglais dans les dictionnaires de prononciation (on aura reconnu la transcription phonémique, telle qu'elle est exigée à l'écrit de l'agrégation d'anglais),

- le système graphique et une interprétation raisonnée d'une lecture possible par un locuteur exprimée à l'aide des symboles phonétiques (c'est la transcription phonétique, plus ou moins « serrée »),

- une interprétation de réalisation concrète d'un locuteur réel (une transcription phonétique) vers une transcription phonémique ou des séquences graphiques (autrement dit de la version : vers la variété d'anglais de référence).

- rien n'interdit toute sorte de "thème », traduction dans une variété donnée d'une séquence de la variété de référence, soit la transposition de séquences graphiques (ou phonémiques) vers l'interprétation phonétique d'une variété d'anglais.

- on peut également construire tout type de conversion entre un système de transcription (qu'elle soit phonémique ou phonétique) fondé sur l'API et tout autre système de transcription (entendu comme représentation graphique à l'aide de signes dont le fonctionnement diverge, plus ou moins, de celui des lettres). A titre d'exemple, on peut 
chercher à convertir les systèmes utilisés dans les dictionnaires de langue et les dictionnaires de prononciation.

Si la transcription est une partie de la métalangue phonologique, elle n'est pas simple ni même complète. Elle doit être considérée comme un métadiscours plus que comme un métalangage. En particulier, la transcription serrée peut fonctionner comme métadiscours. Les diacritiques peuvent ainsi détailler les caractéristiques d'un phone : il est dévoisé, il est apical. S'y donne à voir une théorie des «traits » comme centrale à la description, matérialisée dans les diacritiques. De notre point de vue, «Il n’y a pas de métalangage » doit en fait se lire comme "il n'y a pas de transcription serrée parfaite » (mais il y a du métadiscours, c'est-à-dire de la transcription, plus ou moins affinable). Il n'y a pas davantage de système de translittération parfait où les opérateurs grammaticaux se laisseraient décomposer en une nomenclature universelle et transparente. Il convient de garder à l'esprit que la question du degré de réalisme revient à un questionnement du rapport entre un métadiscours et l'objet langue décrit. Un réalisme strict (c'est-à-dire une figure de vérité) de la transcription phonétique serait sans doute une modalité phonologique du surplomb métalinguistique. Nous ne poserons donc pas ici la question du "réalisme » de la transcription, a fortiori de la transcription large, c'est-à-dire du choix de noter conventionnellement une réalisation par un symbole de l'API plutôt qu'un autre en vertu de ce que ce symbole sert à noter par ailleurs. C'est par exemple la préoccupation de Lilly \& Viel 2001. Il nous semble que cette question gagne à être appréhendée à partir de l'analyse des valeurs formantiques plutôt qu'à la lumière des représentations sur le trapèze de Jones. C'est en tout cas toute la question de la stabilité référentielle (en clair, ce que le phonéticien cherche à décrire) qui est en jeu : associer à un symbole une valeur fixe alors que précisément le symbole phonétique ne vaut pas pour lui-même mais pour ce qu'il donne à représenter dans le système où il fonctionne.

Une fois précisé le statut métadiscursif des deux grands types de transcription, revenons au rôle joué par le symbole phonétique et par les conventions notationnelles.

\section{Représentations et conventions : le statut du symbole phonétique}

Les conventions notationnelles sont indispensables à la prise en charge métalinguistique des niveaux de représentations. L'initiation à la phonologie consiste à dénouer ce noeud gordien où s'entremêlent :

- $\mathrm{i}$ - des niveaux de représentations,

- ii - des degrés de précision dans les niveaux de représentations utilisés,

- iii - des systèmes sémiotiques pour en rendre compte.

Une chance néanmoins : on peut distinguer entre ces systèmes. Nous récapitulons cette situation sous forme d'un tableau:

\begin{tabular}{|l|l|l|l|}
\hline & la réalisation & les symboles & les lettres \\
\hline exemples & $\begin{array}{l}\text { ce que l'on entend à l'initiale de } \\
<y e t>\end{array}$ & $\begin{array}{l}\text { le jod } \\
/ \mathrm{j} / \text { ou }[\mathrm{j}]\end{array}$ & $<\mathrm{j}><\mathrm{J}><\mathrm{j}><\mathrm{J}>$ \\
\hline
\end{tabular}




\begin{tabular}{|l|l|l|l|}
\hline $\begin{array}{l}\text { degré de précision } \\
\text { sémiotique régime }\end{array}$ & $\begin{array}{l}\text { - phonémique } \\
\text { - allophonique }\end{array}$ & $\begin{array}{l}\text {-transcription large } \\
\text { serrée }\end{array}$ & -minuscule \\
\hline $\begin{array}{l}\text { système } \\
\text { sémiotique }\end{array}$ & API & $\begin{array}{l}\text { écriture } \\
\text { alphabétique }\end{array}$ \\
\hline $\begin{array}{l}\text { dénomination? } \\
\text { forme } \\
\text { citationnelle }\end{array}$ & & le jod & le $\mathrm{j}$ \\
\hline
\end{tabular}
de système sémiotique propre, mais uniquement possibilité de construire des métadiscours, dont les colonnes adjacentes pourraient être des illustrations. Nous serions tenté d'évoquer une autre matrice de métadiscours possibles que constitue la visualisation de la parole. Les réalisations peuvent se commenter à partir de leurs valeurs formantiques (voir par exemple les premiers chapitres de Ladefoged 2001).

a distinction absolue et permanente des niveaux d'analyse est une condition de possibilité sine qua non de l'analyse phonologique. De ce point de vue, tout signe tracé en phonologie se doit d'être encadré entre crochets ou barres obliques, comme rappel autonymique élémentaire («ceci est une représentation graphique / phonétique / phonologique »). Nous pourrions dire en résumé que le phonologue peut compter sur un métalexique, qui a ses homonymes, mais où la connotation autonymique des conventions notationnelles a un rôle déterminant.

Nous proposons ensuite une sorte d'analyse métalinguistique de l'objet représenté (la valeur sonore) et des signifiants utilisés pour ce faire (les symboles). On se souvient du choix de l'association phonétique internationale d'utiliser des lettres de l'alphabet roman pour l'alphabet phonétique international et de ses conséquences (accusations d'européanocentrisme et confusions). Nous envisageons quelques équivoques représentationnelles s'agissant du statut des symboles phonétiques. Passée l'erreur initiale d'une simple superposition de valeurs entre lettre et symbole (qui peut superficiellement valoir pour $\mathrm{p}$ ou b en position initiale), deux écueils représentationnels (conceptuels) guettent l'étudiant : considérer le symbole comme une lettre et considérer le symbole comme un dessin.

\section{Le symbole comme lettre}

Lutter contre cette terrible littéralité du symbole peut prendre la forme suivante : faire convertir un système de transcription en un autre (par exemple les conventions du Webster en API ou l'API en SAMPA ${ }^{22}$ ). Plus anecdotiquement, on peut demander aux étudiants d'inventer leur propre système de transcription, par exemple en imposant de créer un diacritique de la sonoritéén. Il nous semble que c'est un des moyens de rendre la «double articulation» du symbole: il a une valeur graphique qui correspond par transposition à un inventaire phonémique donné. Signe parmi d'autres signes (utilisés pour transposer l'inventaire phonémique dans son entier), il est aussi en relation avec ces autres signes graphiques de la transcription, en partie par le jeu des diacritiques. 


\section{Tracer une lettre et dessiner un symbole} celle d'un dessin, c'est-à-dire d'une correspondance bi-univoque signifiant / référent. Des lignes tracées sur le papier se voient reconnaître un statut particulier. Dans le même temps, la perception du symbole phonétique comme un élément irréductiblement spécifique est un obstacle à la lecture entendue comme une activité permettant de composer des relations de combinaison (le B, A - BA). Cette correspondance bi-univoque n'est pas unique, elle est répétable, et dans cette répétition s'hypostasie, se subsume le type sous l'occurrence (je peux retracer le même symbole, dont la signification est bien le renvoi au type, quand bien même il ne serait pas absolument identique à mon premier tracé). La fixité du signifiant est relative, et surtout distinctive. Mes tracés successifs correspondent à un même symbole pour autant que ces différences ne soient pas signifiantes dans le système de conventions en vigueur. Par exemple pour l'API, tant que le symbole ne sera pas confondu avec un autre ${ }^{24}$. Ceci revient à rappeler également qu'une «valeur» phonétique sera interprétée et représentée par un ou plusieurs diacritiques.

\section{Avant et après Kiel : le fétichisme du symbole comme dessin}

Pour les étudiants, la difficulté se matérialise avec le constat que des mêmes symboles, étiquetés "API ", peuvent varier d'un manuel à un autre selon qu'ils ont été publiés avant ou après Kiel [voir la liste des symboles en annexe]. Pour l'enseignant, les réactions face à ce qui est la conséquence de la réforme de l'API sont un symptôme du fétichisme du symbole. Bien que n'affectant qu'un nombre limité de symboles (mais dont les valeurs phonétiques associées sont problématiques pour les étudiants puisqu'elles mettent en jeu la tension et la diphtongaison), cette modification de la convention (à une certaine valeur sera désormais affecté un symbole plutôt qu'un autre) fait figure de révélateur d'un malentendu. La transcription phonémique n'est pas un étalonnage de valeurs comme le serait une mesure physique mais l'affirmation du principe de convertibilité d'un donné langagier en un jeu de conventions où chaque unité de l'inventaire phonologique est représentable. La transcription phonologique est une transposition (ce qui revient à dire qu'elle est transposable à tout autre système de conventions). Ce qui se laisse assez bien convertir en API d'avant ou d'après Kiel.

\section{Interférences sémiotiques}

Nous détaillons deux grands types d'interférences: celles qui affectent les différences entre transcription serrée et transcription large d'une part et celles qui portent sur les propriétés de l'écriture et de la transcription d'autre part.

\section{Interférences entre transcriptions}

Nous nous proposons de décrire ces interférences sémiotiques à partir du triptyque signifiant / signifié / référent ; dessinant dans leurs inter-relations ce que nous désignons comme des «régimes sémiotiques». Manipuler des symboles phonétiques dont la 
signification fluctue selon le type de transcription (et dont le sens même peut varier à proportion) revient à accepter deux « régimes sémiotiques » dont les principes sont contradictoires :

- A un signifiant graphique unique correspond un signifié unique en transcription serrée. Le symbole phonétique note une valeur phonétique et une seule, modulo toute variation diacritique.

- A un signifiant graphique unique correspond en transcription large une classe de référents, les allophones, lorsque le symbole phonétique note un phonème en transcription large.

Non seulement les principes de ces régimes sémiotiques sont contradictoires, mais ils empruntent au même système sémiotique: l'alphabet phonétique international. La transcription serrée ne s'effectue pas seulement à partir d'un jeu de symboles jamais utilisés en transcription large, mais avec les mêmes symboles API (qui sont toujours des symboles API, qu'ils soient ou non utilisés en transcription large). A un régime sémiotique propre (transcription serrée) n'est pas associé un système sémiotique propre ${ }^{25}$ (contrairement à ce qui se passe quand l'API est utilisé pour une transcription large d'une graphie).

\section{Interférences entre écriture et transcriptions}

41 Nous nous bornons à signaler certaines situations paradoxales en terme de processus cognitifs sous-tendant ces praxies :

- La transcription permet de passer de deux régimes sémiotiques (ce que j'entends, ce que j'écris) à quatre (l'oral, l'écrit, la transcription serrée et la transcription large).

- Comparant des transcriptions phonétiques et des transcriptions graphiques (des mots écrits...), les étudiants font le constat, à l'écrit, de l'absence de bi-univocité entre ces systèmes graphiques.

- Le signifiant se dédouble d'un signifiant graphique et d'un signifiant phonologique qui sont potentiellement divergents. (Ainsi deux signifiants phonologiques pour un signifiant graphique dans le cas des homographes paronymes tels que par exemple lead avec le sens de « conduire » ou de « plomb»).

- La transcription conduit à l'abandon de la majuscule (qui peut noter dans certains cas des symboles phonétiques pour d'autres langues que l'anglais ${ }^{26}$.

- Autre interférence sémiotique : la codification de la diphtongue emprunte au code écrit. C'est en un sens un digraphe : deux symboles [e] et [i] comptent pour un seul phonème /ei/.

- Transcrire conduit à des associations contre-intuitives. Le $<\mathrm{c}>$ de car est souvent transcrit $\mathrm{par} / \mathrm{c} /$ dans les transcriptions au lieu de / $\mathrm{k} /$. Dans ces exemples s'opère une transgression de l'orthographe: deux «lettres» (relevant il est vrai de deux systèmes sémiotiques différents) sont comme interchangeables, ce que l'orthographe proscrit.

- Se manifeste la difficulté à saisir les deux niveaux d'analyse et à distinguer entre symbole et lettre.

- La transcription rigoureuse impose la perte de l'allomorphie pour les symboles (dans sa version particulière, «allographie ", où tout signe tracé vaut raisonnablement pour ce qu'il représente, où un jambage retourné ne sera pas signifiant tant qu'on ne confond pas le signe avec un autre). Il n'en va pas de même pour la transcription en symboles phonétiques, où la taille du jambage peut être discriminante, voire où un jambage peut se faire diacritique (le $\mathrm{z}$ avec ou sans barre au milieu). 
- Le recours à un nouveau système sémiotique (l'API) multiplie les risques de confusions entre lettres et symboles et entre symboles phonétiques (symbole du « i » tendu ou relâché). Sans doute faudrait-il introduire ici la notion de parographes (confusables) construit sur le modèle de paronymes, désignant des signifiants graphiques ayant une certaine ressemblance au point d'être confondus. L'activité de transcription suppose la perte de l'allographie, impose une certaine stabilité graphique du signe tracé au risque de la parographie, tant dans la lecture que dans l'écriture, qu'elle soit manuscrite ou électronique 27.

\section{L'usage et la mention}

Outre la question du choix du paradigme désignationnel de la classe des allophones, déjà évoquée, l'analyse phonologique n'échappe pas à des brouillages entre usage et mention. A côté d'effets assez ludiques («l'auteur fait ici une syncope») on est confronté à une difficulté toute particulière pour la mention des formes faibles (et du schwa). La forme citationnelle est la forme pleine; or, c'est la forme réduite que l'on souhaite faire entendre. Cela plaide en faveur d'un " paradoxe performatif » : l'élément langagier résiste à sa désignation dans le moment même de sa profération. Une fois de plus, l'occurrence produite dans le métadiscours ne rend pas justice au type.

\section{Conclusion}

Nous avons constaté (on voit mal comment il en serait autrement) que l'explicitation des catégories phonologiques, leurs illustrations et paradigmes, sont en relation avec le système linguistique dans son entier. En particulier, l'explicitation des inventaires vocaliques met en jeu tout le lexique. L'enseignement de la phonologie, pour «technique» qu'il soit, n'échappe pas aux difficultés de toute métalangue (cas d'homonymie avec la langue courante, confusions possibles entre l'usage et la mention). Esquissons toutefois quelques caractéristiques de la métalangue phonologique qui seraient plus spécifiquement liées au statut particulier de cette discipline.

\section{Hétérogénéité à son objet}

Discours sur une matière qui ne lui est pas homomorphe (ce n'est pas un écrit qui commente de l'écrit mais de l'écrit qui commente de l'oral), la phonologie est hétérogène à son objet. Le métier de phonologue consiste à travailler sur plusieurs niveaux de représentations simultanément (a fortiori dans le cas d'une phonologie graphophonématique). En ce sens, toute phonologie est multilinéaire.

\section{Abstraction}

La métalangue en phonologie est liée à un certain nombre de choix théoriques. Sans même rentrer dans des équivoques de la représentation qui seraient propres à des cadres théoriques particuliers (paliers autosegmentaux, tires, tableaux de la théorie de l'optimalité), la phonologie est le lieu d'une abstraction trop souvent insoupçonnée, contre l'apparente simplicité ou disponibilité des phénomènes au plan sonore. Rappelons cette évidence que savoir prononcer un mot ne met pas à l'abri de la complexité des 
phénomènes en jeu, tout comme être locuteur natif d'une langue ne protège de rien en terme d'équivoque métalinguistique.

\section{Travail sur de l'allophonique}

On doit pouvoir rendre compte, au-delà de la reconnaissance de ses propres tares, des équivoques représentationnelles qu'un métadiscours contribue à instaurer. Il y a des «effets pervers" des métadiscours dont il faut être conscient. Certaines équivoques représentationnelles procèdent d'une série de malentendus au terme desquels il est trop facile de dire que seul l'imbécile regarde le doigt quand le sage montre la lune ${ }^{28}$. Tout ramène à l'occurrence; ce qui montre l'abstraction du type en phonologie. S'y ajoute en enseignement initial l'extrême solidarité des notions linguistiques à transmettre (allophones et phonèmes, transcription serrée et allophonie, graphème et digraphe pour n'en donner que quelques-unes). Les exemples sont susceptibles d'engendrer des effets de paradigme, l'occurrence peut être prise pour le type. Ceci est un autre nom de l'interface phonétique / phonologie. On distingue habituellement entre point de vue onomasiologique (du conçu au produit) et point de vue sémasiologique (du perçu au compris / représenté). La "scission » entre phonétique et phonologique est dans cet entre-deux. Une corrélation des différences d'approche que l'on peut récapituler ainsi :

\begin{tabular}{|l|l|}
\hline bottom-up & top down \\
\hline phonétique & phonologique \\
\hline onomasiologique & sémasiologique \\
\hline
\end{tabular}

\section{Intérêt}

La phonologie est une bonne propédeutique qui insiste sur la précision (dans le tracé des symboles) et sur la rigueur (dans les conventions notationnelles comme dans les raisonnements accentuels). Si cette contribution ne donne qu'un aperçu de la manière dont on imprime un imaginaire linguistique de langue seconde, en l'occurrence à des francophones, elle essaie de situer les difficultés peut-être plus spécifiques à l'enseignement, et partant à la description, de la phonologie. Elle insiste sur la dimension scripturale de la métalangue en phonologie et sur son caractère sténographique. Elle permet de faire retour, réflexivement mais pas définitivement, sur le métalinguistique. Nous en rappelons quelques propositions :

- L'activité métalinguistique révèle la postulation sous-jacente de cette interpénétration entre la conceptualisation théorique, les niveaux de représentation et les choix terminologiques et désignationnels.

- Au-delà des contradictions performatives, observables pour les formes faibles, il faut prendre en considération les effets discursifs en phonologie entre le type et l'occurrence. On peut multiplier les occurrences sans donner le type en cadeau.

- La référence des concepts métalinguistiques pourrait fort bien ne pas être uniforme dans le temps. Le concept métalinguistique travaille. Tout l'écart est entre la définition et son 
exploitation, comme l'exprimait un slogan publicitaire jadis utilisé pour un jeu de réflexion, A minute to understand, a lifetime to master.

\section{BIBLIOGRAPHIE}

Ballier, N., (1997). Les écoles françaises de linguistique anglaise, thèse de doctorat nouveau régime non-publiée, sous la direction de J.J. Lecercle, Université de Paris X-Nanterre.

Ballier, N., (à paraître), Le statut « cognitif » des unités phonologiques, le cas des voyelles de l'anglais. In J. Albrespit \& H. Portine (Eds.) Actes du séminaire TELANCO, Bordeaux : Presses Universitaires de Bordeaux.

Durand J. \& Boë L.-J. (2001). L'opposition consonne/voyelle : avatar historique ou dichotomie opératoire ?, in Orbis Supplementa 17, Métalangage et terminologie linguistique (pp. 785-810). Leuven : Peeters.

Durand, J. (2001). La transcription phonétique de l'anglais : problèmes théoriques et pratiques. In W. Rotgé (dir.), Agrégation externe d'anglais : mode d'emploi, (pp. 181-219). Paris : Ellipses.

Huart, R. (1997). La simplicité trompeuse de la terminologie en anglais oral, LINX, n 36, Paris XNanterre : Publidix.

International Phonetic Association (1999). The Handbook of the International Phonetic Association. Cambridge: Cambridge University Press.

Ladefoged, P. (2001). Vowels and Consonants. London: Blackwell.

Ladusaw, W.A. \& Pullum, G.K. (1996). Phonetic Symbol Guide. Chicago: University of Chicago Press.

Lilly, R. \& Viel, M. (2001). Représentation et réalité en phonologie anglaise. in Etudes anglaises T 54, $\mathrm{n}^{\circ} 4$ (387-400), Paris : Klincksieck / Didier-Erudition.

Martinet, A. (1958). Savoir pourquoi et pour qui l'on transcrit. In La linguistique synchronique (pp. 168-173) Paris : PUF.

Milner, J.-Cl. (1989). Introduction à une science du langage. Paris : Seuil.

Trévise, A. (1996). Réflexion, réflexivité et acquisition des langues, in AILE, 8, (pp. 5-40).

Trévise, A. (2001). Métadiscours explicatifs ou métalangue de bois ? Quelques exemples tirés d'exposés de candidats à l'agrégation interne d'anglais, in Orbis Supplementa 17, Métalangage et terminologie linguistique (pp. 739-750). Leuven: Peeters, 2001.

Wells, J. \& House, J., (1995). The Sounds of the IPA. London: UCL.

Wells, J. (1982). Accents of English. 3 volumes. Cambridge: Cambridge University Press.

Wells, J. (2000). Longman Pronouncing Dictionary. London : Longman.

CORPUS

Une lecture attentive des ouvrages suivants est à l'origine de quelques-uns de nos questionnements, quand bien même les cadres théoriques de référence seraient effectivement différents et assumés comme tels. 
Deschamps A. et al. (2000). Manuel de phonologie de l'anglais, Paris : Didier Erudition / CNED.

Duchet, J.L. (1990). Code de l'anglais oral. Paris : Ophrys.

Ginésy, M. (1995). Mémento de phonétique anglaise. Paris : Nathan.

Ginésy, M. (2001). Phonétique et phonologie de l'anglais. Paris : Ellipses.

Huart, R. (2002). Grammaire orale de l'anglais. Paris : Ophrys.

Lilly, R. \& Viel, M. (1998). La prononciation de l'anglais. Règles phonologiques et exercices de transcription. Paris : Hachette Supérieur.

Lilly, R. \& Viel, M. (1998). Initiation raisonnée à la phonétique de l'anglais. Paris : Hachette Supérieur.

Montreuil, J.-P. (2001). Phonologie de l'anglais, Rennes : Presses universitaires de Rennes.

\section{NOTES}

1. Voir la liste en annexe. Il ne saurait s'agir d'une critique, même implicite, de ces manuels, puisque s'y intéresser constitue précisément une preuve de leur intérêt.

2. On ne fera pas l'économie d'une réflexion sur le système décrit. On trouvera, chemin faisant, non pas une réécriture de la division phonétique/phonologie mais une exploration de cette interface à travers une réflexion sur les interférences des niveaux de représentation.

3. La question des jointures ne sera pas abordée.

4. En toute rigueur, il conviendrait de définir cette notion, si prégnante et si polymorphe en phonologie, de représentation. Minimalement, nous ne l'entendons pas comme le produit d'un dispositif théorique (au sens d'une représentation d'un phonème en traits) mais, naïvement, comme la manière dont un individu appréhende conceptuellement les notions.

5. . Pour une théorisation de cette lecture de l'insu, voir le cinquième chapitre de notre thèse (Ballier 1997).

6. C'est la Grammaire orale de l'anglais qui fait figure d'exception, mais il ne s'agit pas ici de dresser un palmarès. Le paradoxe de notre présentation d'un ordre pédagogique qui n'est pas le plus rationnel ne nous échappe pas.

7. La prononciation majoritaire de "I shall focus..." dans les oraux peut faire l'objet d'une mise au point.

8. Voir l'article de Durand \& Boë 2001 pour un panorama et un questionnement de la notion.

9. Voire ce qui est parfois noté /C/ et sans compter certaines des règles "mixtes" de l'école de Guierre qui posent des régularités sur des séquences graphiques dans des contextes phonologiques.

10. La mauvaise prononciation peut servir de révélateur des équivoques représentationnelles. Une prononciation en « sim-peul » atteste sans doute de l'isosyllabicité et la voyelle épenthétique signale que le principe de la consonne syllabique n'est pas acquis.

11. Sans parler du faible rendement de la phonologisation de ces allophones historiques et d'alternances comme breath breathing où le contexte est responsable de la sonorité.

12. Pour une analyse plus poussée du statut théorique de ce TH- comme trace énonciative, voir le cinquième chapitre de notre thèse (Ballier 1997).

13. Sans compter la peur de la prononciation, la manière dont le francophone est stigmatisé par ses réalisations et ce que ce cliché sonore véhicule, y compris dans le vécu scolaire (voir la réponse insolente dans Les Quatre cents coups de l'élève qui suit les explications du professeur "Je sais pas, moi monsieur, mettre la langue où vous dites").

14. Il suffit pour s'en convaincre, si besoin était, de comparer les transcriptions d'étudiants à partir de dictée phonétique ou d'exercices de transcription à partir d'un texte. 
15. L'autre implication est l'anglais langue seconde, où des communautés de locuteurs ont en partage des règles grapho-phonématiques parfois communes malgré des langues maternelles différentes.

16. A commencer par le phonème lui-même, qui n'existe pas sous forme isolée, mais en systèmes de phonèmes.

17. Les voyelles peuvent également être appréhendées à partir des ensembles lexicaux (lexical sets ) décrits dans Wells 1982. Nous ne les problématisons pas ici, au-delà de l'observation préliminaire que ces inventaires de "divergences potentielles » entre variétés d'anglais ne se ramènent pas à une liste des phonèmes au sens strict, puisque l'on y distingue la classe de BATH et la classe de START. Nous revenons plus longuement sur ces questions dans un article à paraître.

18. Voir les zones plus ou moins grisées des "nuages" représentant les voyelles dans la préface du dictionnaire de Wells.

19. Nous allégeons les notations autant que possible, sans redoubler toutes les citations ou formes citationnelles de guillemets.

20. Nous empruntons cette notion à Jean-Claude Anscombre. Nous renvoyons au quatrième chapitre de notre thèse pour une exploitation théorique du caractère processif de certains métatermes en linguistique énonciative.

21. Nous ramenons à une opposition bipolaire ce qui peut être vu comme un continuum vers du plus en plus détaillé. Voir Durand 2001.

22. SAM Phonetic Alphabet, codification des symboles phonétiques en caractères ASCII. Voir la page web de Wells: WELLS, J.C., SAMPA, UCL http://www.phon.ucl.ac.uk/home/sampa/ english.htm

23. Pèse ici une équivoque, où l'étudiant peut confondre la sonore et la sonnante.

24. L'API s'est doté d'un manuel détaillant les symboles en vigueur (International Phonetic Association 1999). Il existe de plus un manuel détaillant tous les symboles utilisés en phonétique, y compris les tracés des symboles et l'historique de leur utilisation (Ladusaw \& Pullum 1996).

25. L'exercice d'identification du statut d'une transcription (phonétique ou phonologique) ne va pas de soi quant aux argumentations qui sont données. Il n'est pas rare de s'entendre dire que l'on a affaire à une transcription phonétique parce qu'il y a des diacritiques; ce qui revient à occulter le diacritique des monophtongues longues utilisé aussi en transcription large.

26. Sans parler de la notation des archiphonèmes.

27. John Wells remarquait récemment sur la liste de diffusion PHONET que l'introduction d'un nouveau jeu de polices (Lucida Sans Unicode) augmentait les confusions possibles entre les symboles API. Pour un relevé des équivoques des symboles phonétiques non-romains, voir sa page http://www.phon.ucl.ac.uk/home/wells/confusables.htm

28. Nous passerons sur l'inadéquation d'une telle scénographie. The guide on the side, not the sage on the stage, disent-ils.

\section{RÉSUMÉS}

En phonologie, l'activité métalinguistique est indissociable des niveaux de représentations. A partir de quelques problèmes concrets, on envisage les questions théoriques propres au rapport entre l'objet langue envisagé sous sa matérialité sonore et les discours construits pour en rendre 
compte au niveau segmental. On montre ainsi que l'activité de représentation passe par une déconstruction des représentations existantes, y compris les plus fondatrices (ce qu'est une consonne, une voyelle ou une syllabe). La transcription, par le recours à des symboles phonétiques qui sont des systèmes sémiotiques dont l'inventaire et la syntaxe diffèrent de l'alphabet romain, induit des interférences sémiotiques qui sont examinées. Cette contribution insiste sur la dimension scripturale de la métalangue en phonologie et sur son caractère "sténographique" (Milner).

This paper questions some of the possible transcriptions of English including the Kiel reform of the IPA. It is argued that the graphical (ie spelling), phonetic and phonological transcriptions encapsulate a metalinguistic representation of the segmental level. Based on the analysis of some of the problems encountered by (French) EFL learners with transcription, this paper addresses some of the metalinguistic issues at stake in the very act of transcription. Some of the semiotic consequences of the use and syntax of phonetic symbols in transcriptions are assessed, hinting at a potential underlying phonological representation of English based on spelling for literate EFL learners.

INDEX

Mots-clés : API, phonème, SAMPA, symbole phonétique, transcription phonétique

\section{AUTEUR}

\section{NICOLAS BALLIER}

Université de Rouen, ERAC/CETAS (EA 2125) 